\title{
Field Deployments of a Self-Contained Subsea Platform for Acoustic Monitoring of the Environment around Marine Renewable Energy Structures
}

\author{
B.J. Williamson, B.E. Scott, J.J. Waggitt \\ Institute of Biological and Environmental Sciences \\ University of Aberdeen \\ Aberdeen, UK \\ b.williamson@abdn.ac.uk
}

\author{
C. Hall, E. Armstrong \\ Marine Scotland Science \\ Aberdeen, UK
}

\author{
$\mathrm{Ph}$. Blondel \\ Department of Physics \\ University of Bath \\ Bath, UK \\ p.blondel@bath.ac.uk
}

\author{
P.S. Bell \\ National Oceanography Centre \\ Liverpool, UK
}

\begin{abstract}
The drive towards sustainable energy has seen rapid development of marine renewable energy devices, and current efforts are focusing on wave and tidal stream energy. The NERC/DEFRA collaboration FLOWBEC-4D (Flow, Water column \& Benthic Ecology 4D) is addressing the lack of knowledge of the environmental and ecological effects of installing and operating large arrays of wave and tidal energy devices. The FLOWBEC sonar platform combines a number of instruments to record information at a range of physical and multi-trophic levels. Data are recorded at a resolution of several measurements per second, for durations of 2 weeks to capture an entire spring-neap tidal cycle. An upward-facing multifrequency Simrad EK60 echosounder $(38,120$ and $200 \mathrm{kHz})$ is synchronized with an upward-facing Imagenex $837 \mathrm{~B}$ Delta $\mathrm{T}$ multibeam sonar $\left(120^{\circ} \times 20^{\circ}\right.$ beamwidth, $\left.260 \mathrm{kHz}\right)$ aligned with the tidal flow. An ADV is used for local current measurements and a fluorometer is used to measure chlorophyll (as a proxy for plankton) and turbidity. The platform is self-contained with no cables or anchors, facilitating rapid deployment and recovery in highenergy sites and flexibility in allowing baseline data to be gathered.
\end{abstract}

Five 2-week deployments were completed in 2012 and 2013 at wave and tidal energy sites, both in the presence and absence of renewable energy structures. These surveys were conducted at the European Marine Energy Centre, Orkney, UK. Algorithms for noise removal, target detection and target tracking have been written using a combination of LabVIEW, MATLAB and Echoview. Target morphology, behavior and frequency response are used to aid target classification, with concurrent shore-based seabird observations used to ground truth the acoustic data. Using this information, the depth preference and interactions of birds, fish schools and marine mammals with renewable energy structures can be tracked. Seabird and mammal dive profiles, predator-prey interactions and the effect of hydrodynamic processes during foraging events throughout the water column can also be analyzed. These datasets offer insights into how fish, seabirds and marine mammals successfully forage within dynamic marine habitats and also whether individuals face collision risks with tidal stream turbines.

Measurements from the subsea platform are complemented by 3D hydrodynamic model data and concurrent shore-based marine X-band radar. This range of concurrent fine-scale information across physical and trophic levels will improve our understanding of how the fine-scale physical influence of currents, waves and turbulence at tidal and wave energy sites affect the behavior of marine wildlife, and how tidal and wave energy devices might alter the behavior of such wildlife. Together, the results from these deployments increase our environmental understanding of the physical and ecological effects of installing and operating marine renewable energy devices. These results can be used to guide marine spatial planning, device design, licensing and operation, as individual devices are scaled up to arrays and new sites are considered. The combination of our current technology and analytical approach can help to de-risk the licensing process by providing a higher level of certainty about the behavior of a range of mobile marine species in high energy environments. It is likely that this approach will lead to greater mechanistic understanding of how and why mobile predators use these high energy areas for foraging. If a fuller understanding and quantification can be achieved at single demonstration scales, and these are found to be similar, then the predictive power of the outcomes might lead to a wider strategic approach to monitoring and possibly lead to a reduction in the level of monitoring required at each commercial site.

Keywords- Marine renewable energy, environmental monitoring, collision risk, remote sensing, multibeam sonar, echosounder, fish, marine mammals, seabirds, predator-prey

\section{INTRODUCTION}

Little is known of the environmental and ecological effects of installing and operating wave and tidal stream marine renewable energy devices (MREDs) [1]. The NERC/DEFRA collaboration FLOWBEC-4D (Flow, Water column and 
Benthic Ecology 4-D) is investigating the potential effects of MREDs at different test sites around the UK, in particular at the European Marine Energy Centre (EMEC) in Orkney (Scotland). The project aims to understand how currents, waves and turbulence at wave and tidal energy sites may influence the behavior of marine wildlife, and how MREDs might alter the behavior of such wildlife as single devices are scaled up to arrays. Mobile predator and prey use of highenergy sites is being investigated to identify and quantify which type of habitats (depth of water column, speed of tides, etc.) predators predictably use in these areas for foraging, and to assess collision risk.

Trends and predator-prey interactions in these sites are known to occur over a variety of temporal and spatial scales [2] requiring data to be captured at a high temporal resolution (several measurements a second) but also for entire springneap tidal cycles (2 weeks). Sampling at different positions within these wave and energy sites is also required, to understand the use of habitats by different species and to assess the effect of the presence / absence of MREDs.

Regulators need to know with a high degree of certainty whether tidal and wave devices will affect the population level of marine species, but measuring population level changes is a long term and large spatial range issue. An approach which can rapidly and accurately identify and quantify any changes in individual behaviour, within a species, brought about specifically by renewable development, can allow the quantification of what those impacts will be at the population level [3].

Although boat surveys can provide high-resolution survey coverage along a track, it is not logistically feasible to monitor a high-energy site continuously at high-resolution for an entire 14-day tidal cycle. The effects of wind, waves and tide reduce positional accuracy such that boat surveys are not able to monitor small-scale interactions of individual targets with MREDs, and the cost of long duration surveys is high. Surface moorings, such as instrumented buoys and surface platforms can reduce cost and increase survey duration, but taking highresolution measurements of the entire water column and measuring the interactions of wildlife with MREDs is limited from an unstable surface platform in these high-energy sites [4]. In the case of both boats and moored surface platforms, there is also the risk of their presence at the surface affecting the species being studied (e.g. birds, fish and marine mammals) $[5,6]$.

Mounting instruments on the MRED of interest provides a stable mounting and simplifies power and data requirements. The interactions of fish with tidal turbines have been imaged using cameras but visibility (turbidity and illumination) limits both the range and survey time of a visual approach [7]. Acoustic instruments mounted on the MRED are adversely affected by turbulence from the MRED itself which can mask the presence and interactions of wildlife [8]. An independent platform allows the instruments to be positioned adjacent to the MRED looking at the MRED from a short distance, enabling the interactions of wildlife to be imaged but also allowing baseline studies to be conducted under similar conditions in an area free from MREDs. This was the approach chosen for the FLOWBEC project.

\section{Methodology}

The FLOWBEC project combines data from the deployment of an upward-facing subsea sonar platform with shore-based bird observations, shore-based marine X-band radar surveys of wave and current data [9] and detailed 3D modeling of the flow and water column.

The FLOWBEC upward-facing sonar platform allows the

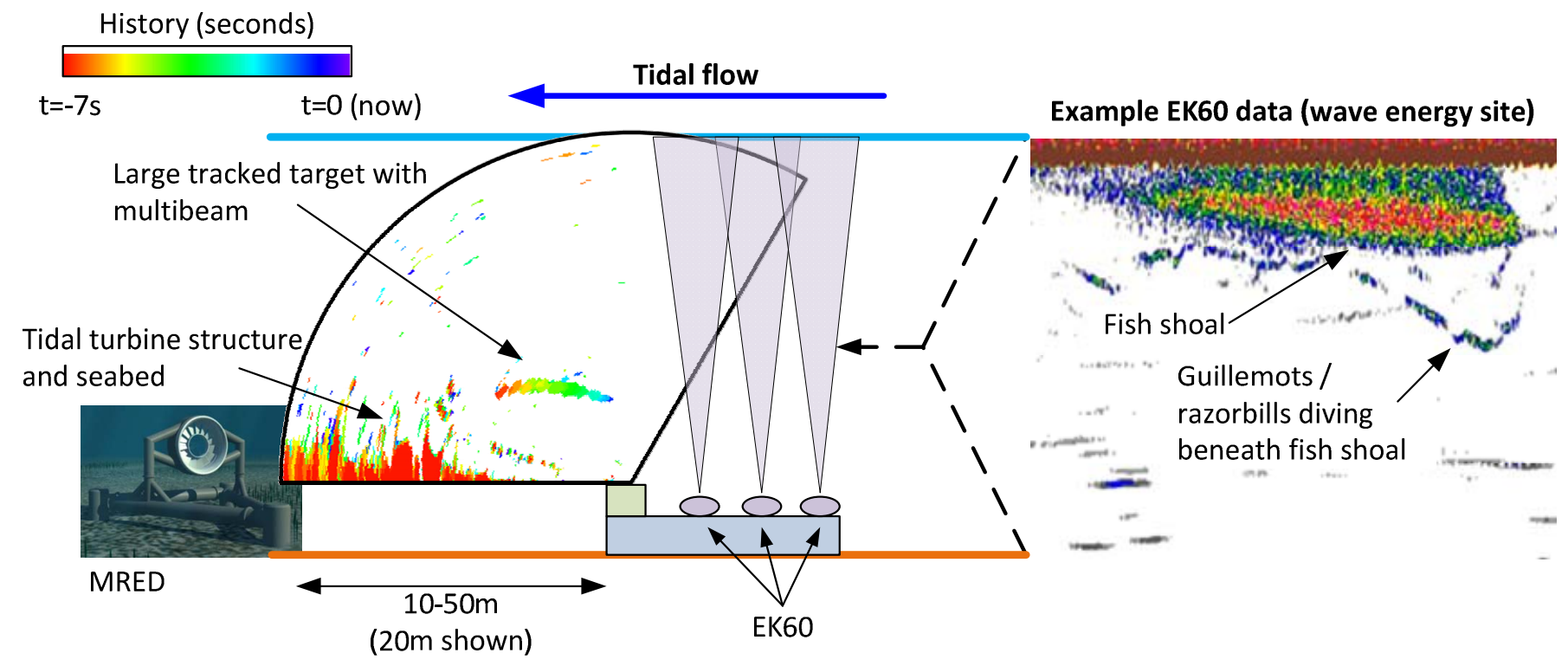

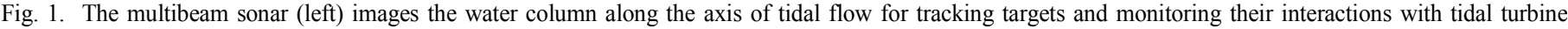

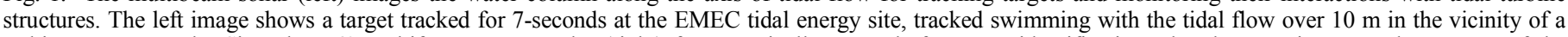

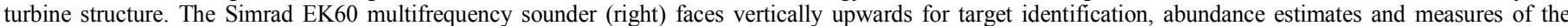

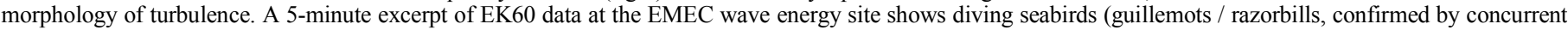
shore-based observations) feeding on the fish shoal. 
interaction of fish, diving seabirds and marine mammals with MREDs to be imaged, and the acoustic environment analyzed as shown in Fig. 1. The platform combines an Imagenex 837B Delta $\mathrm{T}$ multibeam sonar $(260 \mathrm{kHz})$ pinging at several frames per second for target tracking, identification and behavioral analysis, synchronized with a Simrad EK60 multifrequency echosounder (38, 120 and $200 \mathrm{kHz}$ ) used for target identification, abundance estimates, measures of plankton and the morphology of turbulence [10].

The multibeam sonar (MBES) is aligned with its $120^{\circ}$ swath orientated parallel to the tidal flow, and inclined so that the outer beams are parallel to the seabed to include the MRED within the swath. Using this orientation, diving seabirds can be detected above water by the shore-based radar and bird observer, before being tracked underwater in the tidal flow, and the interactions of fish, seabirds and marine mammals with the MRED can be monitored.

The Delta T MBES images a wide swath of $120^{\circ}$ by $20^{\circ}$, with 120,240 or 480 beams, 500 range bins and at repetition rates of up to $20 \mathrm{pings} / \mathrm{second}$. Its range can be adjusted from 0.5 to $100 \mathrm{~m}$ and all parameters (range, gain and ping scheduling) can be controlled programmatically via TCP commands in real-time during operation. The Delta T MBES was selected for its low cost and power consumption (typically $<10 \mathrm{~W}$ ), with similar Imagenex Delta $\mathrm{T}$ models having already been used successfully for a variety of applications [11]. The MBES measures the backscattering strengths (in $\mathrm{dB}$ ) of all targets, relative to a source level of 190 dB re.1 $1 \mathrm{~Pa} @ 1 \mathrm{~m}$ (Patterson, pers. comm., 2012). Pulse lengths vary with the range setting (e.g. $300 \mu$ s at $50 \mathrm{~m}$ range).

The three frequencies of the EK60 echosounder $(38,120$ and $200 \mathrm{kHz}$ ) have $7^{\circ}$ conical beams orientated vertically upwards, facing the water surface. Comparison of scattering strengths at the different frequencies enables identification of fish species, and this echosounder has also been used successfully to examine diving seabirds (e.g. [12]).

An Acoustic Doppler Velocimeter (ADV) provides local current measurements and a fluorometer is used to measure chlorophyll (as a proxy for plankton) and turbidity.

Onboard batteries and data storage for two-week deployments allow an entire spring-neap tidal cycle to be captured. The self-contained seabed platform can be positioned close to the MRED to be investigated allowing the interactions of wildlife to be imaged, but also allowing baseline studies to be conducted under similar conditions in an area free from MREDs or prior to MRED installation.

\section{RESULTS}

Five 2-week deployments have been completed at wave and tidal energy sites at EMEC in Orkney (UK). Deployments were conducted during the seabird breeding season which peaks in summer months [13]. Deployments adjacent to MRE structures (10-20m vicinity) and at 'control' sites in areas free from MREDs were carried out to assess the effect of the presence / absence of these devices.
A suite of Matlab scripts has been written to check consistency of the MBES acoustic measurements. Imaging pulse lengths are set before deployment but checked throughout, as they affect how much energy is put into the water, and therefore how much energy should be scattered back and how it will be interpreted when comparing acoustic variations over entire deployments. Pulse repetition rates are also analyzed to detect any problems with synchronization between instruments; this was for example used to confirm the 2012 repetition rate had to be decreased for the 2013 deployments, to avoid regular acoustic interference between the MBES and EK60 instruments. Finally, individual beam/range cells are analyzed as a function of time, and for each deployment (Fig. 2). This allows identification of expected strong returns, in this case from the turbine, its base, and the seabed around it, or from the sea surface if/when within range. Other beam/range cells might also show relatively higher values over the entire deployment: they might correspond to systematic artefacts or preferential occupation by potential targets (which will then be revealed in the next steps, e.g. target-tracking over time).

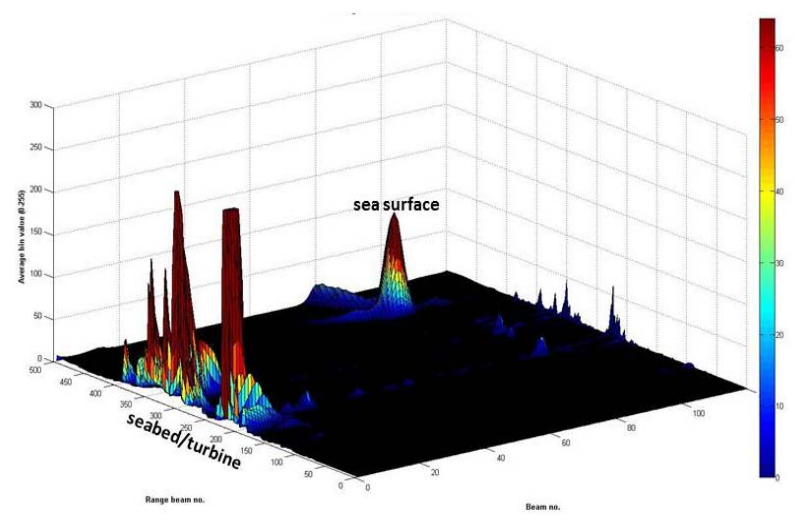

Fig. 2. MBES backscatter (color-coded) is represented as a function of beam and range, to identify areas of consistently high or low returns. The example presented here (Fall of Warness 2013 deployment next to the tidal turbine) clearly shows the high returns of the seabed and turbine, lower returns from the range/beam cells corresponding to the sea surface (1 more spread out as the sea surface will move up and down with the tides, and be smoother or rougher), and much lower returns for some mid-water ranges and orientations, which might correspond to either artefacts or individual targets preferentially moving at these depths.

The acoustic energy scattered back from the sea surface, from any MRED and from individual targets is a good indicator of different processes. Comparisons between sites show clear tidal and diurnal variations (Fig. 3), with higher overall backscatter from study areas with an MRED (Fig. 3, top) than in similar areas without MRED (Fig. 3, bottom). Automatic sea-surface detection algorithms are used to assess these variations within different domains of interest, e.g.: sea surface and its immediate vicinity, giving a proxy measurement of turbulence, backscatter from the MRED itself (which can then be masked out during later processing, or analyzed for targets very close to the MRED) and variations in backscatter at different water depths or areas (which will correspond to individual targets). 

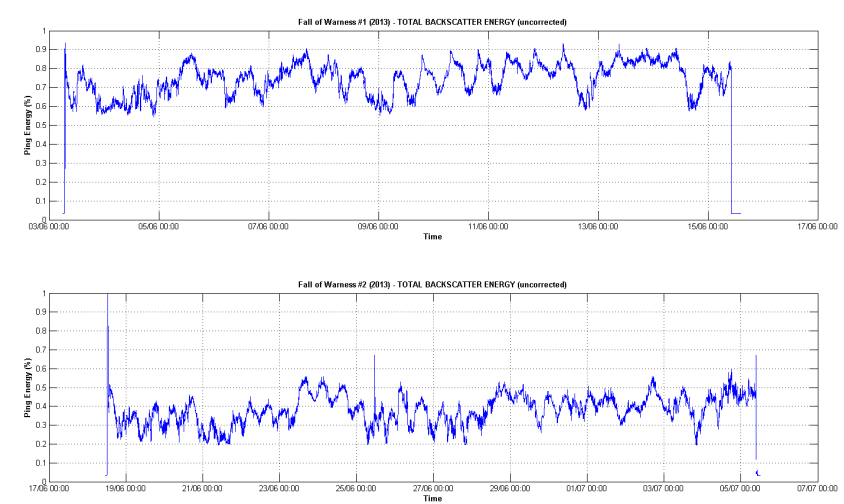

Fig. 3. Total backscatter variations can be analyzed over an entire deployment and compared with similar areas. The example shown here corresponds to the Fall of Warness tidal turbine (top) and control area without turbine (bottom). The sharp changes at the beginning and end of each plot correspond to recovery and deployment, when the MBES is still pinging. Tidal and diurnal variations are clearly visible. Effects from turbine reflections, adding to the overall level, are also clearly visible.

Algorithms for noise removal, target detection and tracking have been written. Fig. 4 shows an example fish shoal tracked using the multibeam within a few meters of the Atlantis AK-1000 tidal turbine structure (shaded in green) at the EMEC tidal site. The turbine blades were not present during this deployment and their expected radius is outlined with a dashed green line.
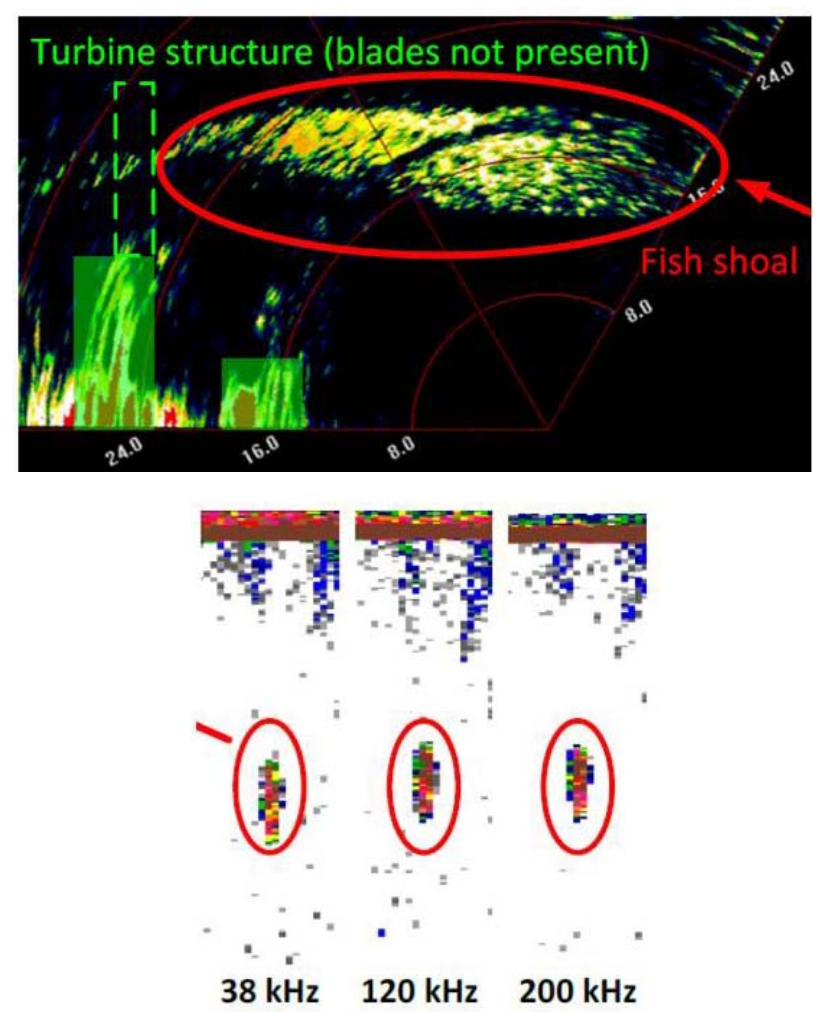

Fig. 4The multibeam swath shows a large fish shoal, tracked over 12-seconds. The turbine structure is outlined in green. The turbine blades were not fitted but their expected radius is outlined in dashed-green. The same target is detectable using the EK60 (shown below), where the frequency response can be used to aid target identification [14].

It is possible to estimate collision risk and the impact of the turbine structure on tracked targets by considering the vertical distribution of targets and what percentage are likely to encounter the turbine structure. Fig. 5 shows the vertical distribution of all tracked targets for a 14-day survey adjacent to a tidal turbine structure. The water column up to a height of $21 \mathrm{~m}$ above the seabed is shown. The turbine structure is shaded in green (to scale) and the dashed outline indicates the expected radius of the blades (blades not present during this survey).

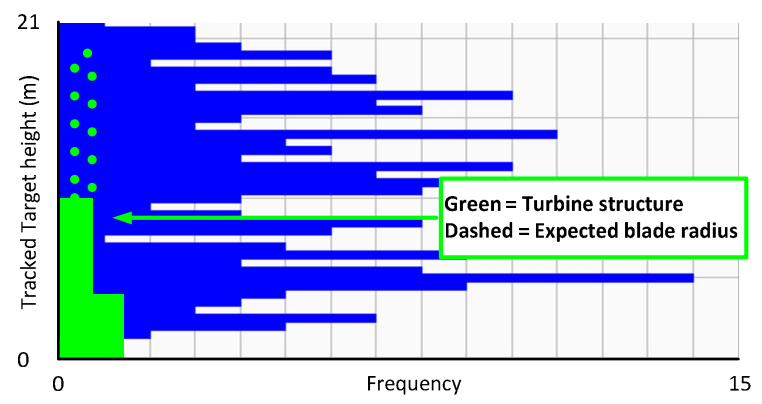

Fig.5. The vertical distribution of tracked targets in the lower $21 \mathrm{~m}$ of the water column over a 14-day survey adjacent to a MRE structure. Many targets are at a height where they are likely to encounter the turbine structure (or blades when present).

\section{DISCUSSION}

The next step is target classification to guide species identification and to allow analysis by time, tide and space for a specific category of targets. Target classification is possible using a variety of methods. The morphology (size, shape, intensity, number of targets per frame, target separation) and behavior (velocity, velocity relative to water column, directionality, vertical distribution and inter-target interaction) can be observed using the multibeam, and classification performed by defining ranges for the various parameters.

Fig. 6 shows four example raw multibeam scans from a deployment adjacent to a tidal turbine structure showing a large shoal of fish, an individual target, a smaller, more densely packed shoal of fish (possibly a different species) and a diving seabird.

Target classification is also possible using multifrequency analysis from the EK60 echosounder data [14]. For fish, the known frequency response of different fish species can be used to identify pelagic and demersal species, and to train software to pick out and track a range of different shoaling / feeding behaviors using the EK60 for identification and the MBES for tracking. The fish shoal in Fig. 4 was also shown in the EK60 echogram for each of the three frequencies. 


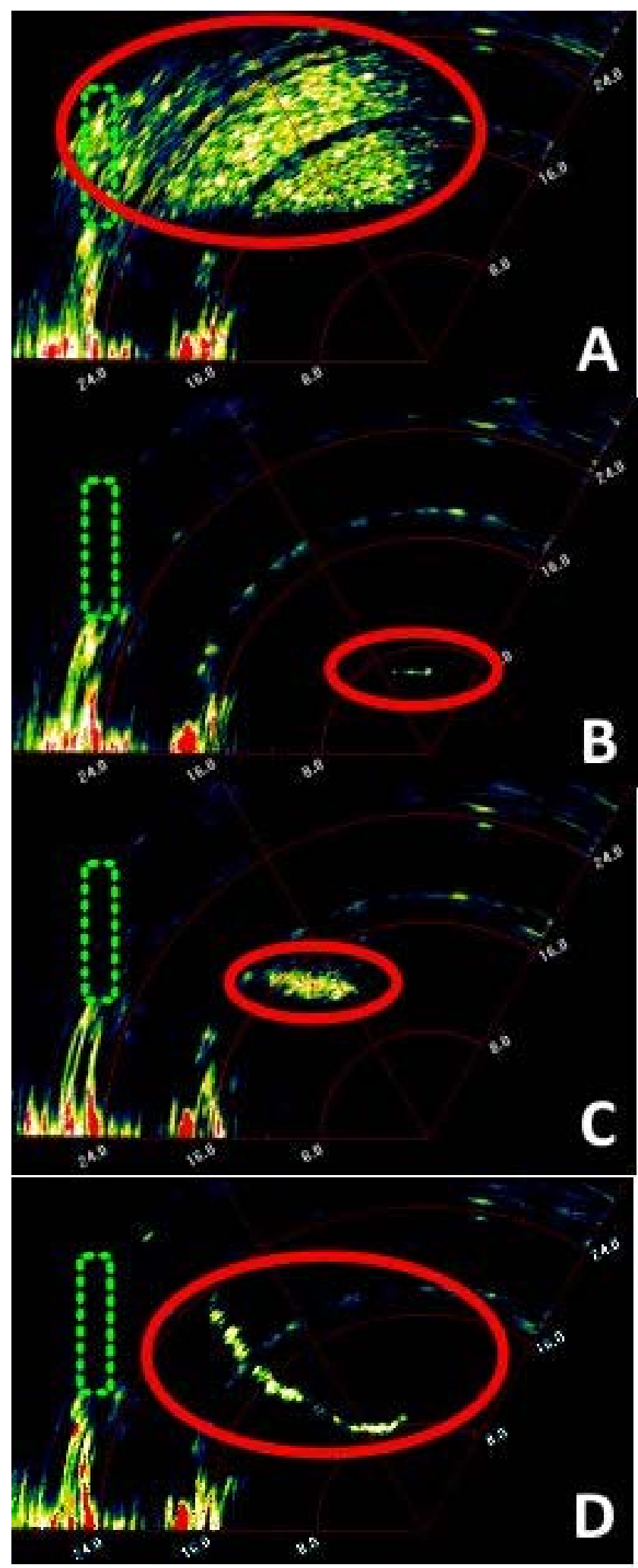

Fig. 6. Four examples of different target types in the vicinity of a tidal turbine structure shown from raw $40 \mathrm{~m}$ range multibeam data averaged over several seconds. The turbine structure is shaded in green, and the expected blade radius is outlined by a dashed green line (blades not present for this survey). Image A shows a large shoal of fish, B shows a small individual target passing through the mid-water, $\mathrm{C}$ shows a denser shoal of fish (possibly a different species) and D shows a diving seabird.
The shore-based wildlife observations are used for ground truthing, particularly for identifying seabird species on the multibeam by their distinctive dive behavior. A subset of shore-based bird observations can be used to first ground-truth acoustic detection of diving seabirds in both sonar instruments, and second to use the known identification of species to 'train' software to pick out different species. The software can then be tested with the remaining shore-based observations.

The outcome of the tracking analysis will allow the environmental effect of MREDs to be explored using the distribution of targets (plankton, fish, birds, marine mammals) and predator-prey interactions with time, tide and space, where space includes vertical use of the water column, and horizontal distribution around the wave and tidal sites, and how all of this changes with the presence and absence of MREDs. The vertical habitat preferences of these ecological groups and collision risks can also be evaluated by looking at spatial overlap with MREDs, and collision risk predicted by looking at the overlap with conditions favoured for MREDs.

\section{CONCLUSIONS}

Increasing commitments to renewable energy in short timescales have seen rapid development of marine renewable energy sources and devices. Little is known of the general effects of installing and operating MREDs, at all depths and in all environments. The FLOWBEC project aims to address the challenge of monitoring a significant portion of animal activity, biological and physical dynamics within the water column and at the sea surface near MREDs, using below-thewater instruments like sonars and above-the-water sensors like radar.

The technology and analytical approach developed in FLOWBEC are currently the only subsurface system to continuously capture fine-scale (several measurements a second, sub-metric spatial resolution) data over a wide range of both physical and multi-trophic levels (plankton, zooplankton, fish, seabirds and mammals) over time periods which encompass day and night differences as well as full spring / neap tidal cycles.

The Imagenex 837B Delta $\mathrm{T}$ multibeam sonar provides high resolution information on a variety of targets in the water column around MREDs. The combined use of an EK60 multifrequency echosounder enables the identification of fish species and has the potential for the identification of seabirds and marine mammals. Fish, marine mammals and diving seabirds can all be tracked during their interactions with MREDs, above water and below water. Acoustic measurements are being analyzed as a function of time, tide, waves, modeled data and shore-based wildlife observations and marine X-band radar to understand the hydrodynamic habitat preference of various functional ecological groups (benthos, plankton, fish, birds and marine mammals) and how individual species may use preferred flow conditions.

Techniques for analyzing the raw data and statistical modeling are being refined, such that the combination of the technology and the analysis will ultimately provide an 
affordable way to measure interactions of marine wildlife in high-energy locations and around MREDs. This combination of our current technology and analytical approach can help to de-risk the licensing process by providing a higher level of certainty about the behavior of a range of mobile marine species in high-energy environments.

It is likely that this approach will lead to greater mechanistic understanding of how and why mobile predators use these high-energy areas for foraging. If a fuller understanding and quantification can be achieved at single demonstration scales, then the predictive power of the outcomes might lead to a wider strategic approach to monitoring and possibly lead to a reduction in the level of monitoring required at each commercial site.

\section{ACKNOWLEDGMENTS}

We would like to acknowledge the technical support of D. Mackay (Hydro Products Ltd., UK) and J. Patterson (Imagenex Technology Corp., Canada) with the multibeam sonar, C. Hall, E. Armstrong, N. Collie, P. Copland, J. Hunter, B. Ritchie, C. Stewart and colleagues at Marine Scotland Science, UK for integration on the FLOWBEC platform, P. Frith and P. Reddish (University of Bath, UK) and colleagues at the European Marine Energy Centre (EMEC). 3D hydrodynamic model data was kindly provided by colleagues P. Cazenave and R. Torres at Plymouth Marine Laboratory. Part of this work was also presented at the Underwater Acoustics Conference (Rhodes, Greece) in June 2014.

\section{REFERENCES}

[1] Shields, M.A., D.K. Woolf, E.P.M. Grist, S.A. Kerr, A.C. Jackson, R.E. Harris, M.C. Bell, R. Beharie, A. Want, E. Osalusi, S.W. Gibb, and J. Side, Marine renewable energy: The ecological implications of altering the hydrodynamics of the marine environment. Ocean \& Coastal Management, 2011. 54(1): p. 2-9.

[2] Waggitt, J.J. and B.E. Scott, Using a spatial overlap approach to estimate the risk of collisions between deep diving seabirds and tidal stream turbines: A review of potential methods and approaches. Marine Policy, 2014. 44(0): p. 90-97.

[3] Scott, B.E., R. Langton, E. Philpott, and J.J. Waggitt, Seabirds and Marine Renewables: Are we Asking the Right Questions?, in Marine Renewable Energy Technology and Environmental Interactions, M.A. Shields and A.I.L. Payne, Editors. 2014, Springer Netherlands. p. 81-92.

[4] Viehman, H.A., G.B. Zydlewski, J.D. McCleave, and G.J. Staines, Using Hydroacoustics to Understand Fish Presence and Vertical Distribution in a Tidally Dynamic Region Targeted for Energy Extraction. Estuaries and Coasts, 2014: p. 1-12.

[5] Tasker, M.L., P.H. Jones, T. Dixon, and B.F. Blake, Counting Seabirds at Sea from Ships: A Review of Methods Employed and a Suggestion for a Standardized Approach. The Auk, 1984. 101(3): p. 567-577.

[6] Schwemmer, P., B. Mendel, N. Sonntag, V. Dierschke, and S. Garthe, Effects of ship traffic on seabirds in offshore waters: implications for marine conservation and spatial planning. Ecological Applications, 2010. 21(5): p. 1851-1860

[7] Broadhurst, M. and S. Barr, Short Term Temporal Behavioural Responses in Pollack, Pollachius pollachius to Marine Tidal Turbine Devices; a Combined Video and ADCP Doppler Approach. in 9th European Wave and Tidal Energy Conference. Southampton. 2011.

[8] Hastie, G.D., Tracking marine mammals around marine renewable energy devices using active sonar. 2012, SMRU Ltd report URN:12D/328 to the Department of Energy and Climate Change. September 2012 (unpublished).

[9] Bell, P., J. Lawrence, and J. Norris, Determining currents from marine radar data in an extreme current environment at a tidal energy test site. in IEEE International Geoscience and Remote Sensing Symposium (IGARSS 2012). 2012. Munich, Germany: IEEE.

[10] Williamson, B.J. and P. Blondel, Multibeam imaging of the environment around marine renewable energy devices. in 11th European Conference on Underwater Acoustics. 2012. Edinburgh, UK: Institute of Acoustics.

[11] Williamson, B.J., M. Balchin, and W.M. Megill, Towards mapping Nereocystis luetkeana kelp beds: Using the holonomic iROV SeaBiscuit and sonar fusion. in 11th European Conference on Underwater Acoustics. 2012. Edinburgh, UK: Institute of Acoustics.

[12] Benoit-Bird, K.J., K. Kuletz, S. Heppell, N. Jones, and B. Hoover, Active acoustic examination of the diving behavior of murres foraging on patchy prey. Marine Ecology Progress Series, 2011. 443: p. 217-235.

[13] Ashmole, N.P., Seabird ecology and the marine environment. Avian biology, 1971. 1: p. 223-286.

[14] Korneliussen, R.J., The acoustic identification of Atlantic mackerel. ICES Journal of Marine Science, 2010. 67(8): p. 1749-1758. 